\title{
Influence of Fluorination on Single- Molecule Unfolding and Rupture Pathways of a Mechanostable Protein Adhesion Complex
}

Journal Article

Author(s):

Yang, Byeongseon; Liu, Haipei; Liu, Zhaowei; Doenen, Regina; Nash, Michael (D)

Publication date:

2020-12-09

Permanent link:

https://doi.org/10.3929/ethz-b-000459089

Rights / license:

Creative Commons Attribution-NonCommercial-NoDerivatives 4.0 International

Originally published in:

Nano Letters 20(12), https://doi.org/10.1021/acs.nanolett.0c04178 


\title{
Influence of Fluorination on Single-Molecule Unfolding and Rupture Pathways of a Mechanostable Protein Adhesion Complex
}

\author{
Byeongseon Yang, Haipei Liu, Zhaowei Liu, Regina Doenen, and Michael A. Nash*
}

Cite This: Nano Lett. 2020, 20, 8940-8950

Read Online

ABSTRACT: We investigated the influence of fluorination on unfolding and unbinding reaction pathways of a mechanostable protein complex comprising the tandem dyad XModule-Dockerin bound to Cohesin. Using single-molecule atomic force spectroscopy, we mapped the energy landscapes governing the unfolding and unbinding reactions. We then used sense codon suppression to substitute trifluoroleucine in place of canonical leucine globally in XMod-Doc. Although TFL substitution thermally destabilized XMod-Doc, it had little effect on XMod-Doc:Coh binding affinity at equilibrium. When we mechanically dissociated global TFL-substituted XMod-Doc from Coh, we observed the emergence of a new unbinding pathway with a lower energy barrier. Counterintuitively, when fluorination was restricted to Doc, we observed mechano-stabilization of the non-

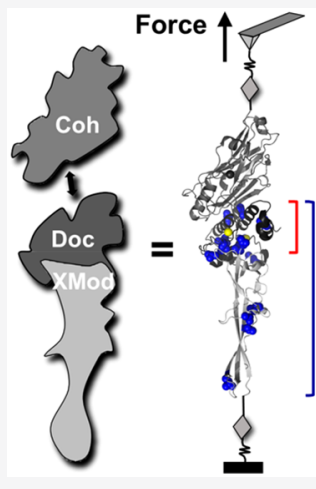

Fluorination by TFL Incorporation fluorinated neighboring XMod domain. This suggests that intramolecular deformation is modulated by fluorination and highlights the differences between equilibrium thermostability and nonequilibrium mechanostability. Future work is poised to investigate fluorination as a means to modulate mechanical properties of synthetic proteins and hydrogels.

KEYWORDS: atomic force microscopy, single-molecule force spectroscopy, protein engineering, fluorine, elastin-like polypeptide, SpyTag/SpyCatcher, molecular deformation

$\mathrm{N}$ on-canonical amino acids (NCAAs) provide new chemical functionality in proteins and can be used to modulate diverse properties including molecular recognition, stability, and activity. ${ }^{1-3}$ In particular, the unique chemical characteristics of fluorine can alter amino acid side chain properties including hydrophobicity, acidity, and reactivity. ${ }^{4-6}$ Incorporation of fluorinated analogues of aliphatic amino acids including leucine (LEU), isoleucine (ILE), and valine (VAL) imparts increased hydrophobicity ${ }^{7-9}$ and is typically marginally disruptive to native protein structure. ${ }^{10}$ Isosteric hydrogen-tofluorine replacement can therefore alter the protein folding energy landscape, in some cases increasing thermodynamic stability but in other cases weakening it. ${ }^{11,12}$ Incorporation of fluorinated amino acids ${ }^{13-15}$ has been shown to influence denaturation temperatures, ${ }^{16-18}$ binding affinity, ${ }^{19}$ enzymatic activity, ${ }^{20-22}$ proteolytic resistance, ${ }^{23,24}$ and folding/aggregation propensity. ${ }^{25}$ Despite a wealth of knowledge on the influence of fluorinated amino acids on protein stability, one current knowledge gap is understanding how fluorination influences protein mechanical properties. To the best of our knowledge, there are no prior studies investigating the influence of fluorination on protein mechanics.

Protein mechanical stability is important in vivo, ${ }^{26,27}$ however the utilization of protein mechanical properties to control engineered synthetic systems is less well established.
Toward this end, mechanically designed polyproteins have been cross-linked into synthetic materials to mimic muscle mechanics, and ligand binding has been used to modulate domain mechanics with concomitant effects on bulk material properties. ${ }^{28,29}$ For engineered systems, fluorination of proteins could provide a useful tool with which to modulate synthetic proteins in the context of mechanotherapeutics, however thus far the impact of fluorination on protein mechanics is not known.

Mechanical stability describes how much tension a folded domain can withstand prior to unfolding, or how much force is required to dissociate a receptor-ligand complex. As prior work has shown, ${ }^{30-33}$ mechanical stability is typically independent from thermostability, and mutant proteins with higher thermal stability are not necessarily more mechanostable. Singlemolecule force spectroscopy (SMFS) ${ }^{34-37}$ with the atomic force microscope (AFM) can be used to stretch single protein

Received: October 19, 2020

Revised: November 8, 2020

Published: November 16, 2020 
A
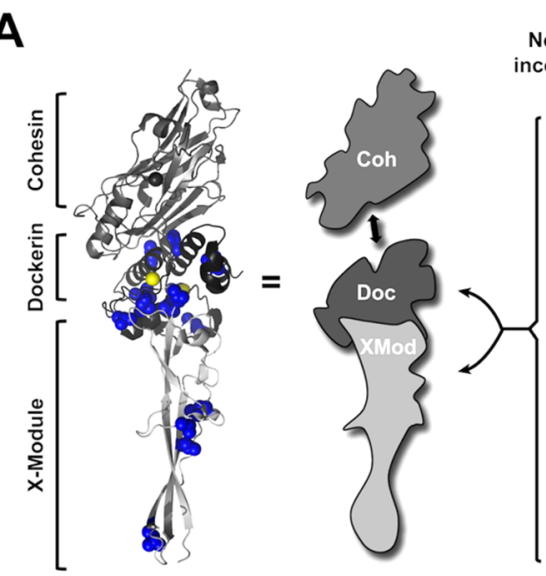

Non-canonical amino acid incorporation into XMod-Doc

Leucine

(LEU)

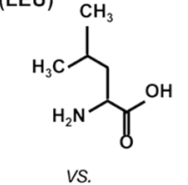

Trifluoro-leucine

(TFL)

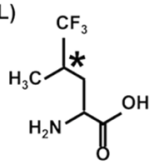

B

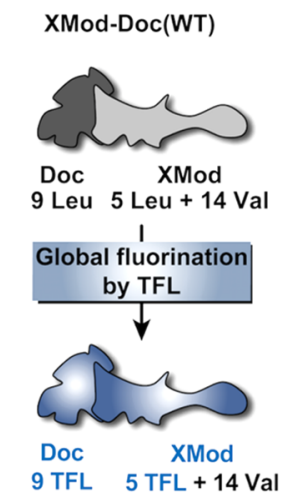

C

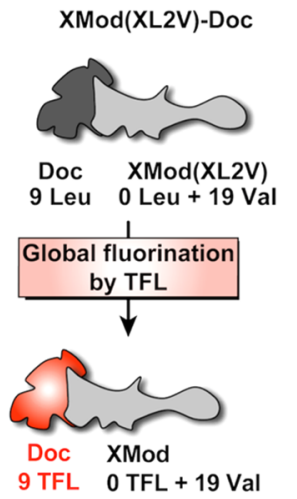

D

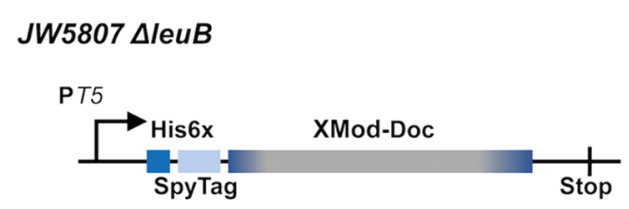

BL21 (DE3)
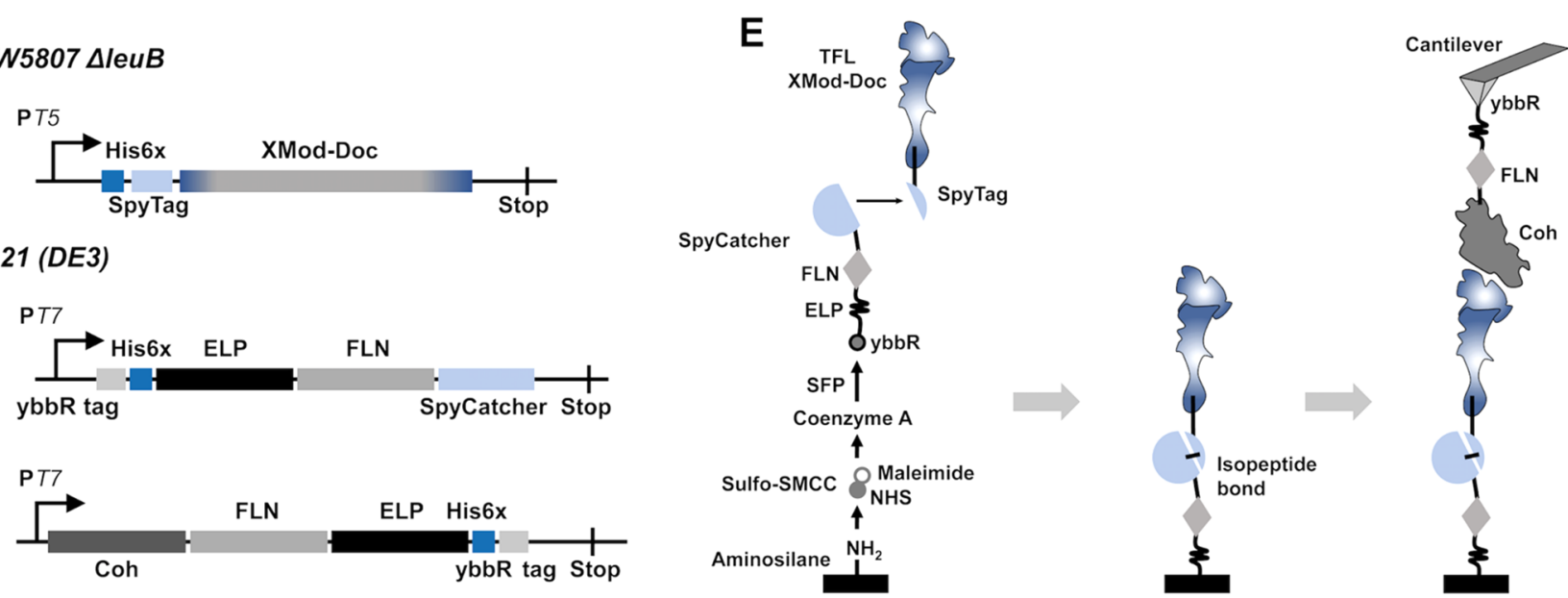

Figure 1. Design of XMod-Doc variants for domain-specific fluorination. (A) Crystal structure of XMod-Doc:Coh showing LEUs (blue), including 2 LEUs at the binding interface. Calcium ions are shown as yellow spheres (PDB 4IU3). XMod-Doc is a single polypeptide chain containing subdomains XMod and Doc that form a high-affinity non-covalent complex with Coh. The TFL structure shows the substituted $-\mathrm{CF}_{3}$ group in place of $-\mathrm{CH}_{3}$ in the side chain of LEU. The chiral center of TFL is indicated with an asterisk. (B) Global TFL incorporation into XMod-Doc (WT) replaced all LEU residues with TFL in both the XMod and Doc subdomains. (C) The mutant XMod(XL2V)-Doc was prepared by mutating all LEU codons in XMod to VAL. Global TFL incorporation resulted in fluorination only in Doc. (D) Gene cassettes for expression of TFLincorporated His-SpyTag-XMod-Doc variants, linker protein ybbR-His-ELP-FLN-SpyCatcher, and Coh-FLN-ELP-His-ybbR. (E) Schematic illustration of surface chemistry and site-specific protein immobilization for AFM-SMFS. FLN, the fourth domain of Dictyostelium discoideum Factin cross-linking filamin.

molecules, quantify intermediate folding states, ${ }^{38,39}$ and elucidate [un] folding energy landscapes while accounting for differences in loading geometry ${ }^{40-44}$ or the presence of dual modes of ligand recognition. ${ }^{45,46}$ The goal of this work was therefore to investigate the role of protein fluorination on nonequilibrium mechanostability, specifically investigating any discrepancies in trends between equilibrium thermodynamic stability and non-equilibrium mechanostability.

As a model protein for this investigation, we chose a mechanostable Dockerin:Cohesin receptor-ligand complex from Ruminococcus flavefaciens ( $R f)$. One binding partner in this complex comprises a tandem dyad of X-module (XMod) and Dockerin (Doc) that forms a non-covalent interaction with the other binding partner, Cohesin ( $\mathrm{Coh}$ ). We denote the complex XMod-Doc:Coh (Figure 1A) where the colon indicates the non-covalent binding interaction. This wellstudied protein pair binds with $\mathrm{nM}$ affinity and exhibits an $\mathrm{O}$ ring ${ }^{47}$ binding patch comprising a hydrophobic center surrounded by hydrophilic polar and charged amino acids. ${ }^{48}$ Prior AFM-SMFS studies on this complex and close homologs ${ }^{49-53}$ have quantified two unfolding/unbinding reaction pathways and demonstrated that XMod stabilizes the Doc:Coh binding interface through an allosteric mechanism governed through contacts between XMod and the distal end of Doc, opposite its interface with Coh. ${ }^{54-56}$ As a consequence, when XMod is folded, the complex is activated and ruptures at high forces, whereas when XMod unfolds, the Doc:Coh interface is significantly weakened. This multidomain polyprotein with interdependency in the mechanical properties therefore provided an opportunity to study the influence of fluorination on both XMod unfolding and Doc:Coh unbinding in the same experiment.

We used TFL as the fluorinated amino acid, where a $-\mathrm{CF}_{3}$ group is substituted for one $-\mathrm{CH}_{3}$ methyl group in LEU. TFL is recognized by endogenous leucyl-tRNA synthetase (LeuRS), enabling quantitative replacement of LEU with TFL without requiring overexpression of endogenous or engineered LeuRS. ${ }^{17,18,57}$ We prepared wild type XMod-Doc (XModDoc (WT)) (Figure 1B) and two mutants where LEU residues in XMod or Doc was replaced with VAL, enabling localized incorporation of TFL into either the XMod or Doc subdomains. We then analyzed WT and mutant protein complexes with canonical LEU or non-canonical TFL-incorporation using mass spectrometry, AFM-SMFS, thermal denaturation differ- 
ential scanning fluorescence (DSF), and isothermal titration calorimetry (ITC) to quantify the effects of hydrogen-tofluorine substitution in the hydrophobic side chain of LEU on mechanical and biophysical behavior.

New Mutant Designs for Localized Fluorination and Attachment Chemistry. We designed XMod-Doc mutants by removing LEU codons from selected regions of the gene cassette encoding XMod-Doc and replacing them with VAL codons. This allowed us to use global LEU sense codon suppression during expression runs in E. coli and achieve localized domain-specific incorporation of TFL into either XMod or Doc. The first new mutant is denoted XMod(XL2V)Doc where all LEU codons from XMod were changed to VAL (Figure 1C). The second mutant was denoted XModDoc(DL2V) where all LEUs from Doc were mutated to VAL. While residue-specific replacement of LEU by TFL resulted in TFL incorporation into both XMod and Doc subdomains for XMod-Doc(WT), TFL incorporation was localized to Doc for XMod(XL2V)-Doc, and to XMod for XMod-Doc(DL2V). The WT and two mutant XMod-Doc proteins were produced using both canonical LEU and noncanonical TFL incorporation and thoroughly characterized to disentangle the influence of TFL on XMod unfolding and Doc:Coh unbinding.

To ensure we only analyzed valid single-molecule interactions, we performed AFM-SMFS with fingerprint domains ${ }^{58-60}$ attached to both the cantilever and surface molecules. We were concerned that TFL incorporation into fingerprint domains would change their unfolding signatures and severely reduce expression yields. To overcome this issue, we developed a novel scheme for site-specific immobilization of TFL-containing proteins for AFM-SMFS (Figure 1D,E). XMod-Doc variants (WT, XL2V, and DL2V) were designed and produced with an N-terminal SpyTag based on the plasmid pQE80L-SpyTag-ELP-SpyTag, a gift from Mark Howarth (Addgene plasmid \#112634; http://n2t.net/ addgene:112634; RRID:Addgene_112634) ${ }^{61}$ (Figure 1D) which forms a spontaneous isopeptide bond with SpyCatcher. $^{62,63}$ We further produced a specialized surface-bound fingerprint protein (Figure 1D) containing SpyCatcher at the C-terminal end. This was fused at the DNA level with the fourth domain of Dictyostelium discoideum F-actin cross-linking filamin (FLN), ${ }^{64}$ which is an established unfolding fingerprint domain with a characteristic intermediate [un] folding state. Inframe with FLN-SpyCatcher on the N-terminal side, we included an elastin-like polypeptide (ELP) as a linker, allowing us to eliminate commonly used poly(ethylene) glycol linkers that are problematic in high-force protein unfolding studies due to mechano-isomerization of PEG that skews contour length analysis. ${ }^{65}$ Finally, a ybbR/6x-histidine tag N-terminal to the ELP allowed for site-specific and covalent immobilization onto coenzyme A (CoA)-functionalized coverglass via ligation by $4^{\prime}$-phosphopantetheinyl transferase (SFP). ${ }^{66}$ Coh meanwhile was cloned as a single construct. The gene cassettes are depicted in Figure 1D, and the surface chemical scheme is shown in Figure 1E.

Incorporation of TFL. For TFL incorporation, we used the leucine auxotrophic strain E. coli $\Delta$ leuB (JW5807 from the Keio collection). ${ }^{67}$ Residue-specific incorporation was carried out using a standard media saturation method. ${ }^{2,68}$ TFL was charged onto leucyl-tRNAs by endogenous LeuRS and cotranslationally introduced into XMod-Doc variants. Coh and SpyCatcher fusion proteins were expressed using a standard strain (BL21) in rich media (no TFL). All proteins were purified by metal ion affinity chromatography and sizeexclusion. WT and variants expressed using LEU or TFL incorporation are denoted with the relevant amino acid appended after the protein abbreviation (e.g., XMod-Doc(WT)-LEU, XMod-Doc(WT)-TFL, XMod(XL2V)-Doc-LEU, XMod(XL2V)-Doc-TFL).

Sodium dodecyl sulfate polyacrylamide gel electrophoresis (SDS-PAGE) clearly showed successful expression and purification of TFL-incorporated XMod-Doc variants (Figure 2A). We measured TFL incorporation by high-resolution liquid chromatography electrospray ionization mass spectrometry (HRMS) and confirmed high yields of $>92 \%$ for XMod$\operatorname{Doc}(\mathrm{WT})$ and $>90 \%$ for XMod(XL2V)-Doc (Figure 2B,C). Each successful TFL incorporation event is expected to increase protein mass by $+54 \mathrm{Da}$ due to the three hydrogento-fluorine substitutions (18 $\left.\mathrm{Da}^{*} 3\right)$. For XMod-Doc(WT)TFL, the most intense peaks in the HRMS spectrum were $30330 \mathrm{Da}$ and $30281 \mathrm{Da}$ (with a mass difference of +54 Da*15 and +54 Da*14 compared to XMod-Doc(WT)-LEU), corresponding to incorporation yields of $100 \%$ and $93 \%$, respectively. For XMod(XL2V)-Doc-TFL, the most intense peaks in the HRMS spectrum were $29942 \mathrm{Da}$ and $29996 \mathrm{Da}$ (with a mass difference of $+54 \mathrm{Da} * 9$ and $+54 \mathrm{Da} * 10$ compared to XMod-Doc(XL2V)-LEU), corresponding to incorporation yields of $90 \%$ and $100 \%$, respectively. XMod-Doc(WT) and variants were also expressed with canonical LEU and characterized by SDS-PAGE and HRMS (Figures S1, S2). The expected molecular weights of Coh and the SpyCatcher fusion were validated by SDS-PAGE (Figure S3).

We tested the Coh-binding ability of XMod-Doc(WT), XMod(XL2V)-Doc, and XMod-Doc(DL2V) under canonical LEU incorporation using analytical size exclusion chromatography. XMod-Doc(WT)-LEU and XMod(XL2V)-Doc-LEU both bound Coh, however, XMod-Doc(DL2V)-LEU did not (Figure S4). Apparently the nine LEU to VAL substitutions in Doc (119 amino acids in total), two of which are located at the hydrophobic center of the Doc:Coh binding interface, were deleterious and eliminated Doc binding ability. We terminated work on DL2V and no further results are reported on that variant. Additionally, we verified the functionality of SpyTag/ SpyCatcher assembly using analytical chromatography (Figure S5).

AFM-SMFS of XMod-Doc(WT)-LEU and -TFL. Next we performed receptor-ligand AFM-SMFS on XMod-Doc(WT)LEU and XMod-Doc(WT)-TFL bound to Coh (Figure 3A). The surface was repeatedly probed with a Coh-modified cantilever tip, resulting in occasional formation of an XModDoc:Coh complex. The cantilever was retracted at constant speed, ELP linkers were stretched and tension built up in the system until sufficiently high forces were reached to unfold the two FLN fingerprint domains in series. Each FLN unfolding event contained an intermediate state, resulting in 4 low-force $(\sim 60 \mathrm{pN})$ unfolding peaks. Data traces were filtered by searching for two $36 \mathrm{~nm}$ contour length increments with intermediate folding states associated with FLN unfolding (Figure 3D,E). FLN unfolding was followed either by rupture of the XMod-Doc:Coh directly (pathway 1 (P1), Figure 3B), or by unfolding of XMod and subsequent rupture of the Doc:Coh binding interface at greatly reduced forces (pathway 2 (P2), Figure 3C).

XMod-Doc:Coh complex rupture events from P1 and P2 pathways were analyzed and plotted as a function of the 

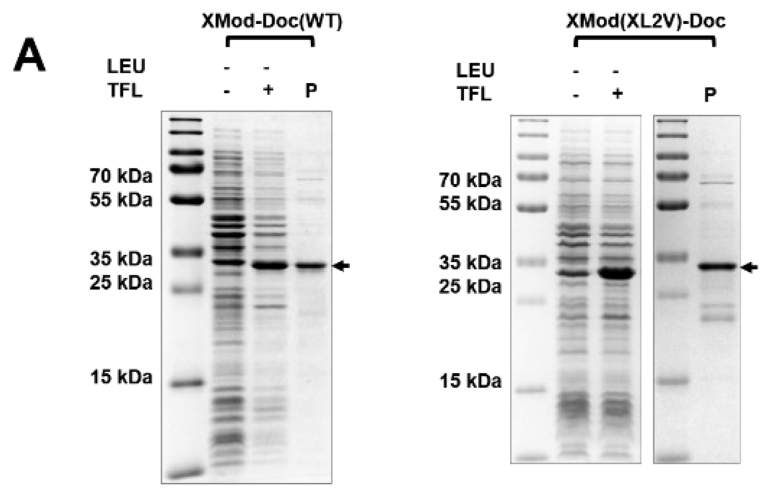

B

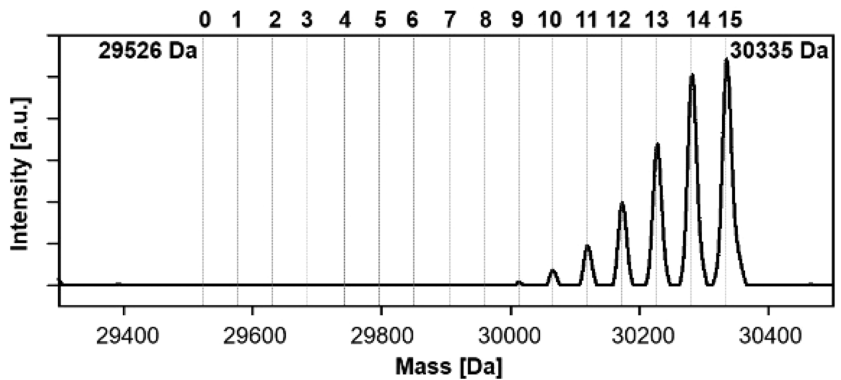

\begin{tabular}{|c|c|c|c|c|}
\hline & & $\begin{array}{c}\text { Theoretical } \\
\text { [Da] }\end{array}$ & $\begin{array}{c}\text { Measured } \\
\text { [Da] }\end{array}$ & $\begin{array}{l}\text { Measured } \Delta m[\mathrm{Da}] \\
\text { / Incorporation Yield }\end{array}$ \\
\hline \multirow{2}{*}{$\begin{array}{l}\text { SpyTag } \\
\text {-XMod- } \\
\text { Doc } \\
\text { (WT) }\end{array}$} & LEU & 29526 & 29527 & \\
\hline & TFL & & $\begin{array}{l}30173(10 \%) \\
30226(19 \%) \\
30281(30 \%) \\
\mathbf{3 0 3 3 5 ( 3 5 \% )}\end{array}$ & $\begin{array}{r}647\left(54 \mathrm{Da}^{*} 12\right) / 80 \% \\
700\left(54 \mathrm{Da}^{*} 13\right) / 87 \% \\
755\left(54 \mathrm{Da}^{*} 14\right) / 93 \% \\
809\left(54 \mathrm{Da}^{*} 15\right) / 100 \%\end{array}$ \\
\hline
\end{tabular}

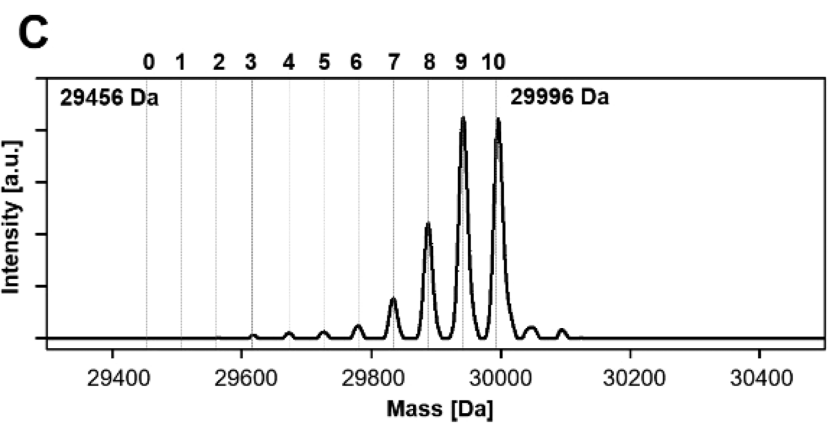

\begin{tabular}{|l|c|c|c|c|}
\hline & & $\begin{array}{c}\text { Theoretical } \\
\text { [Da] }\end{array}$ & $\begin{array}{c}\text { Measured } \\
\text { [Da] }\end{array}$ & $\begin{array}{c}\text { Measured } \boldsymbol{\Delta m} \text { [Da] } \\
\text { / Incorporation Yield }\end{array}$ \\
\hline SpyTag & LEU & 29456 & 29456 & \\
\cline { 2 - 5 } -XMod & TFL & & $29834(6 \%)$ & $378\left(54 \mathrm{Da}^{*} 7\right) / 70 \%$ \\
(XL2V)- & & & $29888(18 \%)$ & $432\left(54 \mathrm{Da}^{*} 8\right) / 80 \%$ \\
Doc & & & $\mathbf{2 9 9 4 2 ( 3 6 \% )}$ & $\mathbf{4 8 6}\left(\mathbf{5 4} \mathrm{Da}^{*} \mathbf{9}\right) / \mathbf{9 0 \%}$ \\
& & & $\mathbf{2 9 9 9 6 ( 3 7 \% )}$ & $\mathbf{5 4 0}\left(\mathbf{5 4} \mathrm{Da}^{*} \mathbf{1 0}\right) / \mathbf{1 0 0} \%$ \\
\hline
\end{tabular}

Figure 2. Residue-specific TFL incorporation into XMod-Doc. (A) SDS-PAGE analysis showing successful expression and purification of XMod-Doc(WT)-TFL and XMod(XL2V)-Doc-TFL. P: purified protein solution. (B) HRMS analysis of XMod-Doc(WT)-TFL. (C) HRMS analysis of XMod(XL2V)-Doc-TFL. Major mass peaks, the percentage of each peak and corresponding incorporation yield of TFL are given in the tables.

loading rate to generate a dynamic force spectrum (Figure 3F) and extract parameters that describe the free energy landscape of the unbinding reaction. Consistent with prior work, ${ }^{49,51}$ XMod-Doc(WT)-LEU:Coh rupture events (Figure 3F, black dots) clearly showed two populations corresponding to a high- force population P1 (470-572 $\mathrm{pN}$ ) and a low-force population P2 (226-273 pN). The same experiment was performed on XMod-Doc(WT)-TFL:Coh complexes. In addition to similar looking P1 and P2 rupture events, we observed a new rupture force population for the TFL-incorporated complex (Figure $3 \mathrm{~F}$, blue dots). This new rupture pathway, denoted P3, was situated at an intermediate force range of $338-432 \mathrm{pN}$ between $\mathrm{P} 1$ and P2. The P3 pathway did not exhibit XMod unfolding prior to complex rupture and was observed as a distinct population in the force histogram (Figure $3 \mathrm{H}$ ).

We analyzed rupture events from P1, P2, and P3 obtained at several pulling speeds $\left(100,400,800,1600\right.$, and $\left.3200 \mathrm{~nm} \mathrm{~s}^{-1}\right)$ and used the phenomenological Bell-Evans $(\mathrm{BE})^{69,70}$ model to extract the energy landscape parameters, $\Delta x$, and $k_{\text {off }}$ (Table $1)$. We found slightly but not significantly lower $\Delta x$ values for P1 $(\Delta x=0.13 \pm 0.01 \mathrm{~nm},-\mathrm{TFL} ; \Delta x=0.15 \pm 0.02 \mathrm{~nm},-\mathrm{LEU}$; Table 1) and significantly lower $\Delta x$ values for P2 rupture events $(\Delta x=0.16 \pm 0.02 \mathrm{~nm},-\mathrm{TFL} ; \Delta x=0.30 \pm 0.06 \mathrm{~nm}$, -LEU; Table 1) for XMod-Doc(WT)-TFL:Coh as compared with XMod-Doc(WT)-LEU:Coh. P3 events unique to TFLincorporated complexes produced slightly higher $\Delta x$ values of $0.17 \pm 0.01 \mathrm{~nm}$ to P1 of TFL-incorporated complexes (Table $1)$. We note that $k_{\text {off }}$ fitting with the Bell-Evans model is not reliable enough to draw quantitative comparisons due to extreme model sensitivity. ${ }^{71}$ Fitted $\Delta x$ values, however, are generally robust. The observed trends in $\Delta x$ for P1 and P2 rupture events indicate that global TFL replacement of LEU in XMod-Doc(WT) resulted in more rigid protein complexes with rupture events exhibiting a steeper loading rate dependency. The appearance of a distinct new P3 unbinding reaction pathway further indicates how TFL-incorporation modulates the energy landscape, in this case by enabling a new unbinding pathway with a lower barrier height.

In addition to unbinding/rupture events, we also analyzed XMod unfolding events obtained from TFL-incorporated and LEU-incorporated XMod-Doc(WT) (Figure 3I-K). XMod$\operatorname{Doc}(\mathrm{WT})$-TFL showed a significant decrease in the unfolding force of XMod (315-431 pN) compared to XMod-Doc(WT)LEU (384-450 pN). $\Delta x$ significantly decreased from $0.23 \pm$ $0.03 \mathrm{~nm}$ for XMod-Doc(WT)-LEU to $0.14 \pm 0.01 \mathrm{~nm}$ for XMod-Doc(WT)-TFL (Table 1). Therefore, we found both for Doc:Coh unbinding as well as XMod unfolding, TFLincorporation resulted in interaction/folding potentials that were shorter and more rigid for TFL-incorporated samples.

AFM-SMFS of Mutant XMod(XL2V)-Doc-LEU and -TFL. Next, we analyzed XL2V mutants under both -LEU and -TFL incorporation (Figure 4A). XL2V allowed TFLincorporation to be localized to Doc while only canonical amino acids were present in XMod. XMod(XL2V)-DocLEU:Coh complexes (Figure 4B, gray) and XMod(XL2V)Doc-TFL:Coh complexes (Figure 4B, red) both showed similar behavior for P1 rupture events with XMod remaining folded and rupture occurring at 488-585 pN (-LEU) and 476$571 \mathrm{pN}$ (-TFL) and also shared similar loading rate dependency with a slightly but not significantly stiffer loading rate dependency (smaller $\Delta x$ ) as compared with XMod$(\mathrm{XL} 2 \mathrm{~V})$-Doc-LEU $(\Delta x=0.16 \pm 0.03 \mathrm{~nm}$, -TFL; $\Delta x=0.18 \pm$ $0.02 \mathrm{~nm},-\mathrm{LEU}$; Table 1). P2 rupture events that occurred following XMod unfolding were in similar force ranges for XMod(XL2V)-Doc-LEU:Coh and XMod(XL2V)-DocTFL:Coh complexes (225-252 pN (-LEU); 219-248 pN $(-\mathrm{TFL}))$. However, $\Delta x$ values of $\mathrm{P} 2$ rupture events showed that, similar to the trend observed in XMod-Doc(WT), TFL- 
A

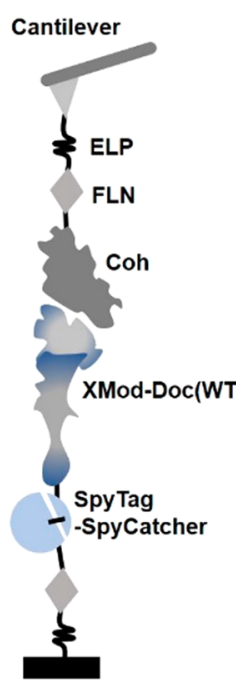

B
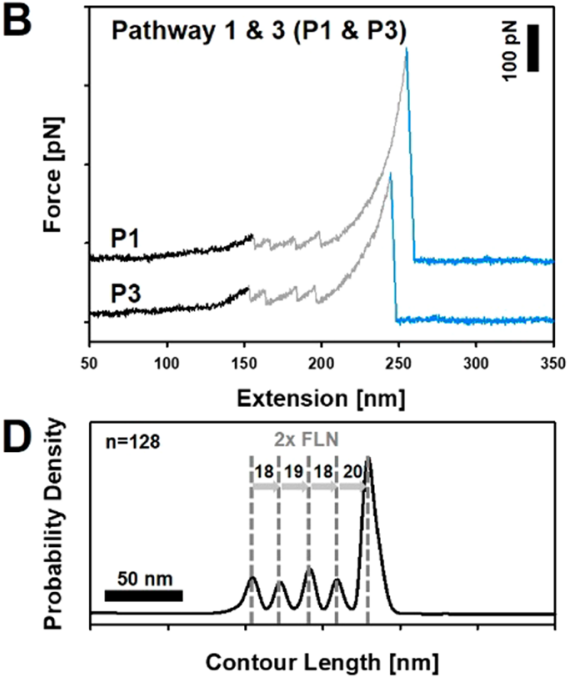
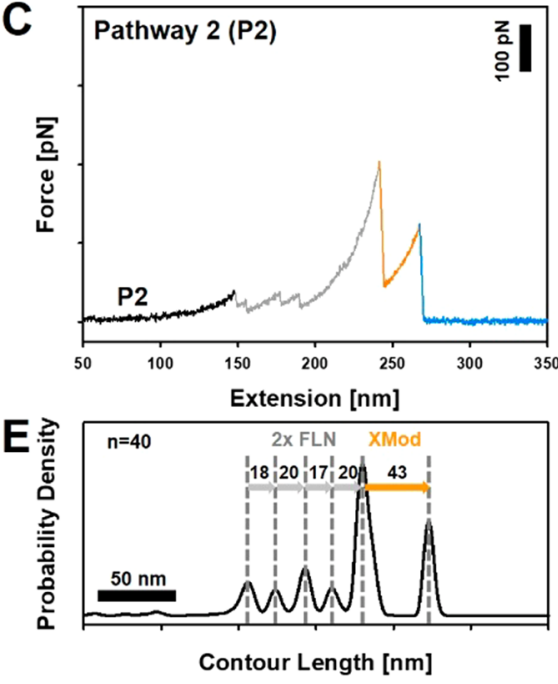

F $\square \begin{aligned} & \text { Coh vs XMod-Doc(WT)-LEU } \\ & \text { Coh vs XMod-Doc(WT)-TFL }\end{aligned}$

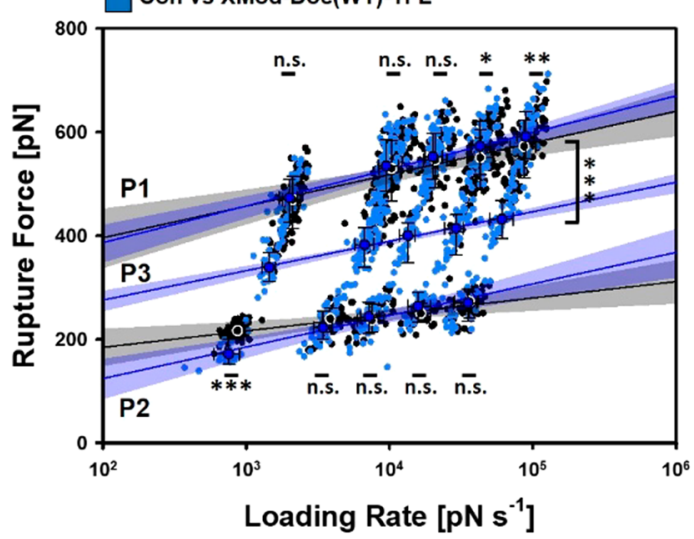

Loading Rate $\left[\mathrm{pN} \mathrm{s}^{-1}\right]$

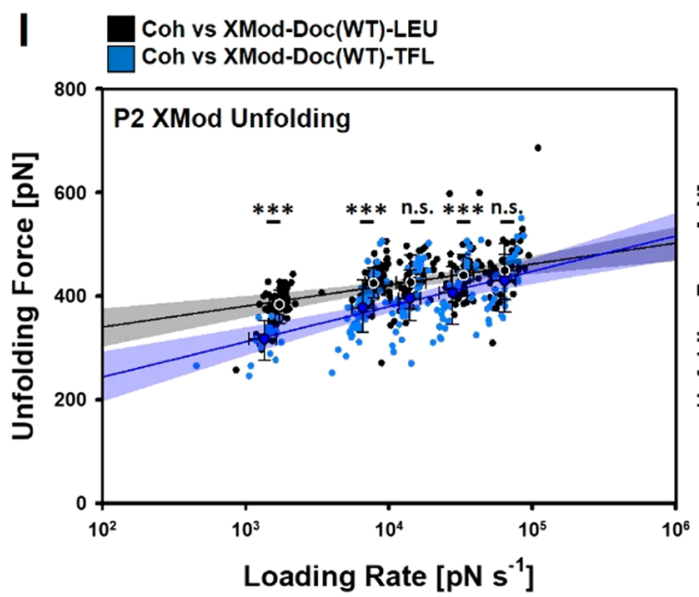

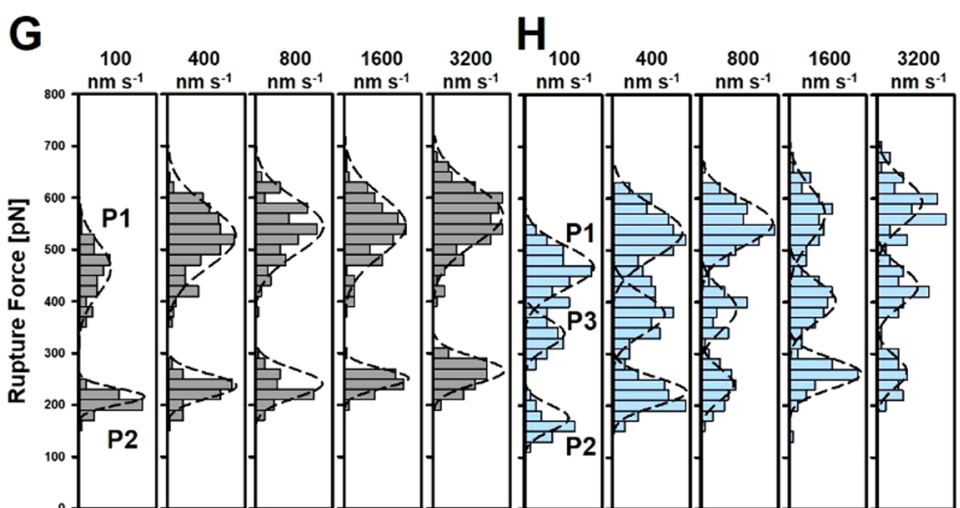

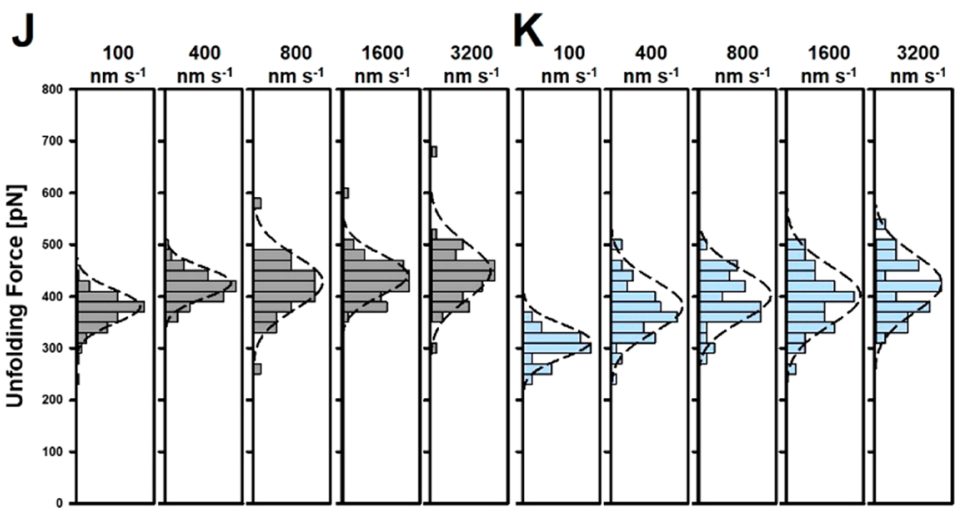

Figure 3. AFM-SMFS of XMod-Doc(WT)-LEU and -TFL. (A) Schematic illustration of experimental configuration. (B) Typical force-extension trace of XMod-Doc:Coh rupture with XMod remaining folded (pathway 1, P1). Unfolding of two FLN fingerprint domains (gray) was used to filter the curves for specific single-molecule interactions. Fingerprint unfolding was followed by complex rupture (blue). (C) Typical forceextension trace showing XMod unfolding followed by Doc:Coh rupture (pathway 2, P2). (D) Contour length histogram of P1 curves ( $n=128)$. (E) Contour length histogram of P2 curves $(n=40)$. Increments between peaks were used for domain assignments to unfolding events. (F) Dynamic force spectra of XMod-Doc(WT)-LEU:Coh (black) and -TFL (blue) complex rupture forces for P1, P2, and P3 events. (G) Histograms of XMod-Doc(WT)-LEU:Coh P1 and P2 rupture events. (H) Histograms of XMod-Doc(WT)-TFL:Coh P1, P2, and P3 rupture events. (I) Dynamic force spectra of XMod unfolding events occurring along P2 pathways. (J) Histograms of XMod unfolding forces from XMod-Doc(WT)LEU:Coh complexes. (K) Histograms of XMod unfolding forces from XMod-Doc(WT)-TFL:Coh complexes. Black and blue circles represent the median rupture force/loading rate at each pulling speed of $100,400,800,1600$, and $3200 \mathrm{~nm} \mathrm{~s}^{-1}$. Error bars are \pm 1 s.d. Solid lines are least square fits to the Bell-Evans model. Shades indicate $95 \%$ confidence intervals. Statistical significance was determined with a t-test: n.s. $p \geq 0.01 ;{ }^{*} p<0.01$; $* * p<0.001$; and $* * * p<0.0001$. 
Table 1. Energy Landscape Parameters of XMod-Doc:Coh Complex Rupture and X-Module Unfolding from AFM-SMFS from Bell-Evans (BE) Model ${ }^{69,70}$

\begin{tabular}{|c|c|c|c|c|}
\hline & & & $\Delta x(\mathrm{~nm})$ & $k_{\text {off }}\left(\mathrm{s}^{-1}\right)$ \\
\hline \multirow[t]{7}{*}{ XMod-Doc(WT) } & LEU & P1 (XMod folded) & $0.15 \pm 0.02$ & $(1.2 \pm 3.1) \times 10^{-6}$ \\
\hline & & P2 (XMod unfolded) & $0.30 \pm 0.06$ & $(1.1 \pm 3.6) \times 10^{-5}$ \\
\hline & & P2 XMod unfolding & $0.23 \pm 0.03$ & $(2.4 \pm 7.3) \times 10^{-8}$ \\
\hline & TFL & P1 (XMod folded) & $0.13 \pm 0.01$ & $(1.2 \pm 1.4) \times 10^{-5}$ \\
\hline & & P2 (XMod unfolded) & $0.16 \pm 0.02$ & $(3.6 \pm 3.0) \times 10^{-2}$ \\
\hline & & P3 (XMod folded) & $0.17 \pm 0.01$ & $(6.1 \pm 4.8) \times 10^{-5}$ \\
\hline & & P2 XMod unfolding & $0.14 \pm 0.01$ & $(8.8 \pm 11.2) \times 10^{-4}$ \\
\hline \multirow[t]{6}{*}{ XMod(XL2V)-Doc } & LEU & P1 (XMod folded) & $0.18 \pm 0.02$ & $(2.7 \pm 6.3) \times 10^{-8}$ \\
\hline & & P2 (XMod unfolded) & $0.40 \pm 0.04$ & $(1.0 \pm 2.1) \times 10^{-7}$ \\
\hline & & P2 XMod unfolding & $0.33 \pm 0.09$ & $(7.6 \pm 66) \times 10^{-12}$ \\
\hline & TFL & P1 (XMod folded) & $0.16 \pm 0.03$ & $(3.0 \pm 9.9) \times 10^{-7}$ \\
\hline & & P2 (XMod unfolded) & $0.26 \pm 0.02$ & $(2.3 \pm 2.1) \times 10^{-4}$ \\
\hline & & P2 XMod unfolding & $0.31 \pm 0.03$ & $(7.0 \pm 42) \times 10^{-9}$ \\
\hline
\end{tabular}

incorporation in $\mathrm{XMod}(\mathrm{XL} 2 \mathrm{~V})$-Doc resulted in binding potentials with a significantly steeper loading rate dependency, which leads to significant difference of $\mathrm{P} 2$ rupture forces (185 $\mathrm{pN}$ (-LEU); $209 \mathrm{pN}$ (-TFL)) at a low pulling speed of 100 $\mathrm{nm} / \mathrm{s}$ (Figure 4B-D; Table 1). Therefore, TFL-incorporation rigidified not only WT complexes but also XL2V complexes in their deformation response. The effect was small but significant for the P2 pathway. The P3 pathway meanwhile that was observed in XMod-Doc(WT)-TFL:Coh complexes was absent in all $\mathrm{XMod}(\mathrm{XL} 2 \mathrm{~V})$-Doc:Coh unbinding reactions. This finding implicates the significant role of XMod in stabilization of Doc:Coh interfaces in the alternative P3 pathway for XModDoc(WT)-TFL.

We next analyzed XMod unfolding events occurring along the P2 rupture pathway for XMod(XL2V)-Doc-TFL and XMod(XL2V)-Doc-LEU at various pulling speeds and extracted energy landscape parameters using BE fitting (Figure $4 \mathrm{E}-\mathrm{G})$. Interestingly, XMod(XL2V)-Doc:Coh complexes showed a small but statistically significant increase XMod mechanical stability under TFL-incorporation in a range of 6$13 \%$ depending on the pulling speeds as compared with LEUincorporation (405-459 $\mathrm{pN}$ (-TFL); 380-430 $\mathrm{pN}$ (-LEU)). While TFL-incorporation rigidified folding potential of WT samples, there is no TFL-incorporation in XMod in XL2V samples and the $\Delta x$ parameter showed no significant change of rigidity in unfolding potential for XL2V samples $(\Delta x=0.31 \pm$ $0.03 \mathrm{~nm},-$ TFL; $\Delta x=0.33 \pm 0.09 \mathrm{~nm},-$ LEU; Table 1). TFL incorporation in Doc therefore significantly increased the force required to unfold the neighboring XMod domain.

We were concerned that small differences in cantilever spring constants could give rise to systematic errors on an absolute force scale. Therefore, to validate this result further, we performed AFM-SMFS experiments to probe XMod(XL2V)-Doc-TFL:Coh and XMod(XL2V)-Doc-LEU:Coh using the same cantilever (Figure $4 \mathrm{H}$ ). Both proteins were immobilized at different positions on the same glass slide, and a single Coh-modified cantilever was used to alternatively pick up and stretch molecules at each location at a pulling speed of $100 \mathrm{~nm} \mathrm{~s}^{-1}$. This experiment eliminated uncertainties based on cantilever calibration error or differences in extension values that arise because molecules on separate cantilevers may be attached at different heights onto the AFM tip. The results confirmed that the XMod was slightly stabilized in XMod(XL2V)-Doc-TFL:Coh complexes and required higher forces (7\% increase) to mechanically unfold.
It could be argued that the higher XMod unfolding forces are observed by a ceiling or biasing effect ${ }^{72}$ of the receptorligand complex. A higher complex stability, one could argue, would result in higher unfolding force distributions for XMod unfolding events simply because the handle used to pull on $\mathrm{XMod}$ is stronger and the unfolding/rupture force distributions are overlapping. However, we verified that P1 and P2 Doc:Coh stability was nearly identical for the XL2V mutants under LEU- and TFL-incorporation (Figure 4B,I). In fact, P1 complex rupture events for XMod(XL2V)-Doc-TFL:Coh were even slightly lower than those for -LEU, therefore, the mechanical strengthening we observe for XMod in -TFL complexes would in fact be underestimated by the biasing effect. The observed differences in XMod stability are therefore not explained by a statistical biasing effect of the complex on the XMod unfolding force distribution. We can speculate that an increase in hydrophobicity within Doc upon TFL-incorporation influenced the contact interface between Doc and XMod. Three LEUs located in the contact interface are well situated in the hydrophobic groove of XMod together with alpha helix $(\alpha 2$, Doc) and they cover $\sim 14 \%$ of calculated contact interface area (Figure S6). The modified contacts could reasonably reroute the force propagation pathway ${ }^{50-52}$ through the molecule, introducing propagation paths with components perpendicular to the pulling axis. Such a scenario would result in an XMod domain that is more effective at distributing the force across its cross section, thereby requiring higher tension to unfold. By contrast, recall that global TFL incorporation into XMod-Doc(WT) significantly decreased the unfolding force of XMod (Figure $3 \mathrm{H}$ ). In-depth analysis by steered molecular dynamics simulation might offer the insight into the mechanism by which fluorination can alter these force propagation pathways.

Bulk Biophysical Properties Stability by ITC and NanoDSF. To investigate the effects of TFL incorporation on equilibrium binding affinity, we performed isothermal titration calorimetry (ITC) (Figure 5A). Analysis of the XModDoc(WT)-LEU:Coh interaction revealed $K_{\mathrm{D}}=60 \mathrm{nM}\left(K_{\mathrm{D}}\right.$ range $( \pm \sigma)=27-121 \mathrm{nM})$, while the XMod-Doc(WT)TFL:Coh interaction showed $K_{\mathrm{D}}=96 \mathrm{nM}\left(K_{\mathrm{D}}\right.$ range $( \pm \sigma)=$ 52-170 nM). Analysis of XMod(XL2V)-Doc-LEU:Coh resulted in $K_{\mathrm{D}}=123 \mathrm{nM}\left(K_{\mathrm{D}}\right.$ range $\left.( \pm \sigma)=70-212 \mathrm{nM}\right)$, while that of XMod(XL2V)-Doc-TFL:Coh showed $K_{\mathrm{D}}=114$ $\mathrm{nM}\left(K_{\mathrm{D}}\right.$ range $\left.( \pm \sigma)=62-201 \mathrm{nM}\right)$. Global LEU to TFL substitutions in the WT sequence may have slightly 

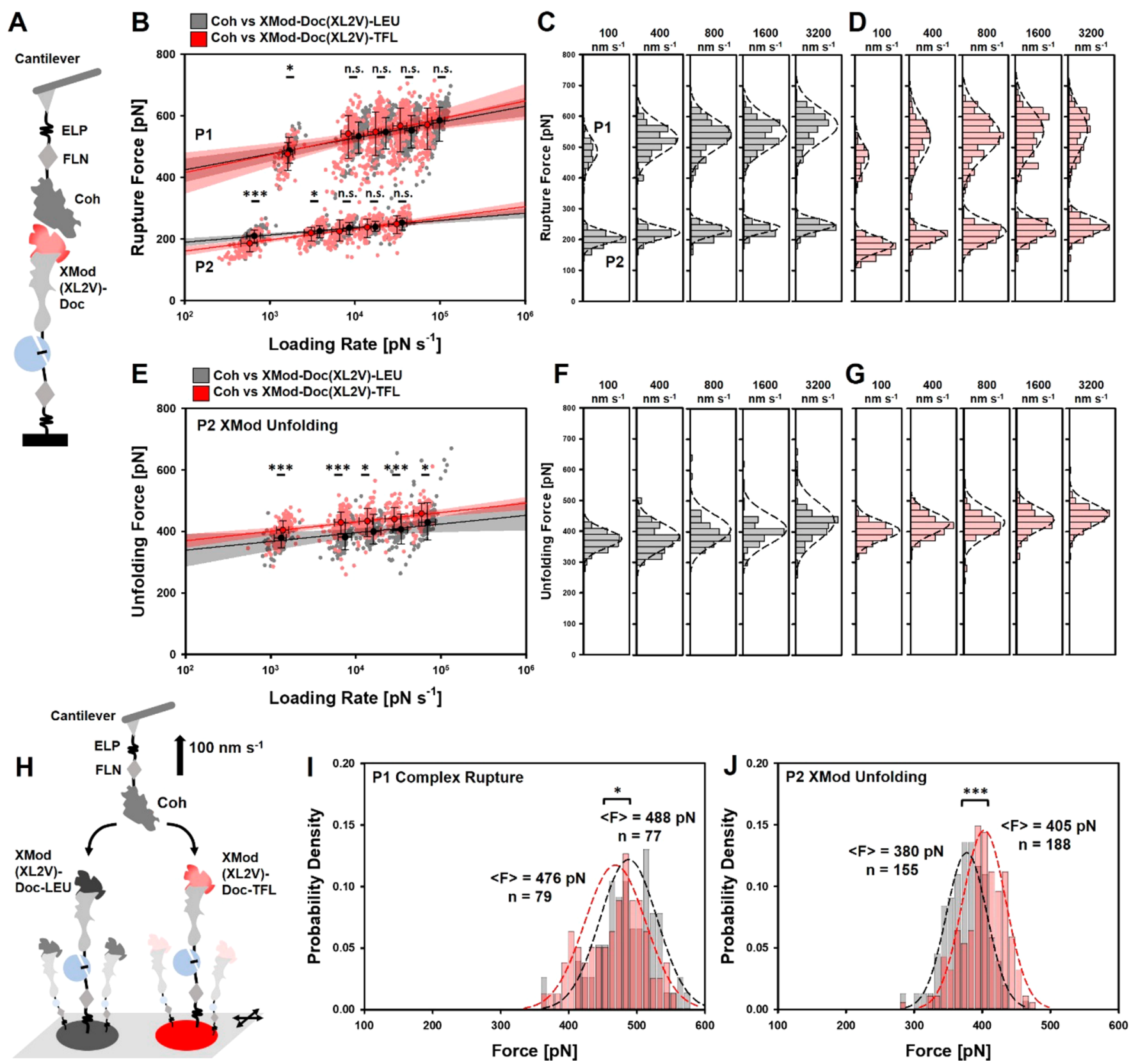

Figure 4. AFM-SMFS of XL2V variants under LEU- and TFL-incorporation. (A) Schematic illustration of experimental configuration, where TFL was incorporated into Doc but not XMod. (B) XMod(XL2V)-Doc-LEU:Coh (gray) and XMod(XL2V)-Doc-TFL:Coh (red) complex rupture events. (C) Histograms of XMod(XL2V)-Doc-LEU:Coh P1 and P2 rupture events at different pulling speeds. (D) Histograms of XMod(XL2V)Doc-TFL:Coh P1 and P2 rupture event at different pulling speeds. (E) Dynamic force spectra of XMod unfolding events. (F) Histograms of XMod unfolding forces from XMod(XL2V)-Doc-LEU:Coh complexes at four pulling speeds. (G) Histograms of XMod unfolding forces from XMod(XL2V)-Doc-TFL:Coh complexes at four pulling speeds. (H) Schematic of the experimental setup for comparative AFM-SMFS of XL2V variants under LEU- and TFL-incorporation. XMod(XL2V)-Doc-LEU and -TFL were immobilized on different spots on the surface. Coh was immobilized at the cantilever tip. The same cantilever was used to alternate between two sample spots and probe each sample intermittently at 100 $\mathrm{nm} \mathrm{s}^{-1}$. (I) Histograms of XMod(XL2V)-Doc-LEU $(n=79)$ and -TFL $(n=77)$ :Coh P1 rupture event. (J) Histograms of XMod(XL2V)-Doc-LEU $(n=155)$ and -TFL $(n=188)$ :Coh P2 XMod unfolding event. Black and red circles represent the median rupture force/loading rate at each pulling speed of $100,400,800,1600$, and $3200 \mathrm{~nm} \mathrm{~s}^{-1}$. All error bars are \pm 1 s.d. Solid lines are least square fits to the Bell-Evans model. Shades indicate $95 \%$ confidence intervals. Statistical significance was determined with a t-test: n.s. $p \geq 0.01 ; * p<0.01 ; * *<0.001$; and $* * * p<0.0001$.

destabilized the interaction at equilibrium; however, given the uncertainties associated with the method these observed differences are not considered significant. Meanwhile, LEU to VAL mutations in Doc (XMod-Doc(DL2V)-LEU) abolished binding ability completely (Figure S4).

Finally, we used differential scanning fluorimetry (DSF) to measure thermal denaturation temperatures (Figure 5B). DSF analysis showed that mutation of Leu to Val in XMod(XL2V)Doc under LEU incorporation/expression decreased the thermal melting temperature by $\sim 5^{\circ} \mathrm{C}$ as compared with XMod-Doc(WT)-LEU. Additionally, TFL incorporation decreased thermal denaturation temperatures of both XModDoc(WT)-TFL and XMod(XL2V)-Doc-TFL by $\sim 8{ }^{\circ} \mathrm{C}$. This significant decrease $\left(15 \%\right.$ on a ${ }^{\circ} \mathrm{C}$ scale $)$ in thermal stability for 
A

XMod-Doc(WT)-LEU $\mathrm{K}_{\mathrm{D}}: 60 \mathrm{nM}$

$\mathrm{K}_{\mathrm{D}}$ range $( \pm \sigma): 27-121 \mathrm{nM}$
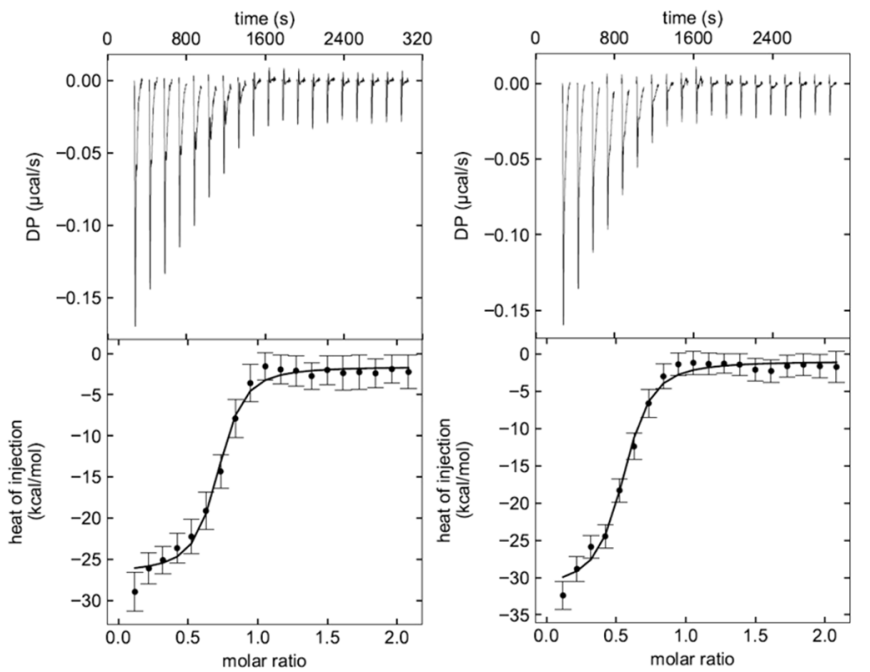

XMod(XL2V)-Doc-LEU

$\mathbf{K}_{\mathrm{D}}: 123 \mathrm{nM}$

$\mathrm{K}_{\mathrm{D}}$ range $( \pm \sigma)$ : $70-212 \mathrm{nM}$
XMod(XL2V)-Doc-TFL

$\mathrm{K}_{\mathrm{D}}: 114 \mathrm{nM}$

$\mathrm{K}_{\mathrm{D}}$ range $( \pm \sigma): 62-201 \mathrm{nM}$
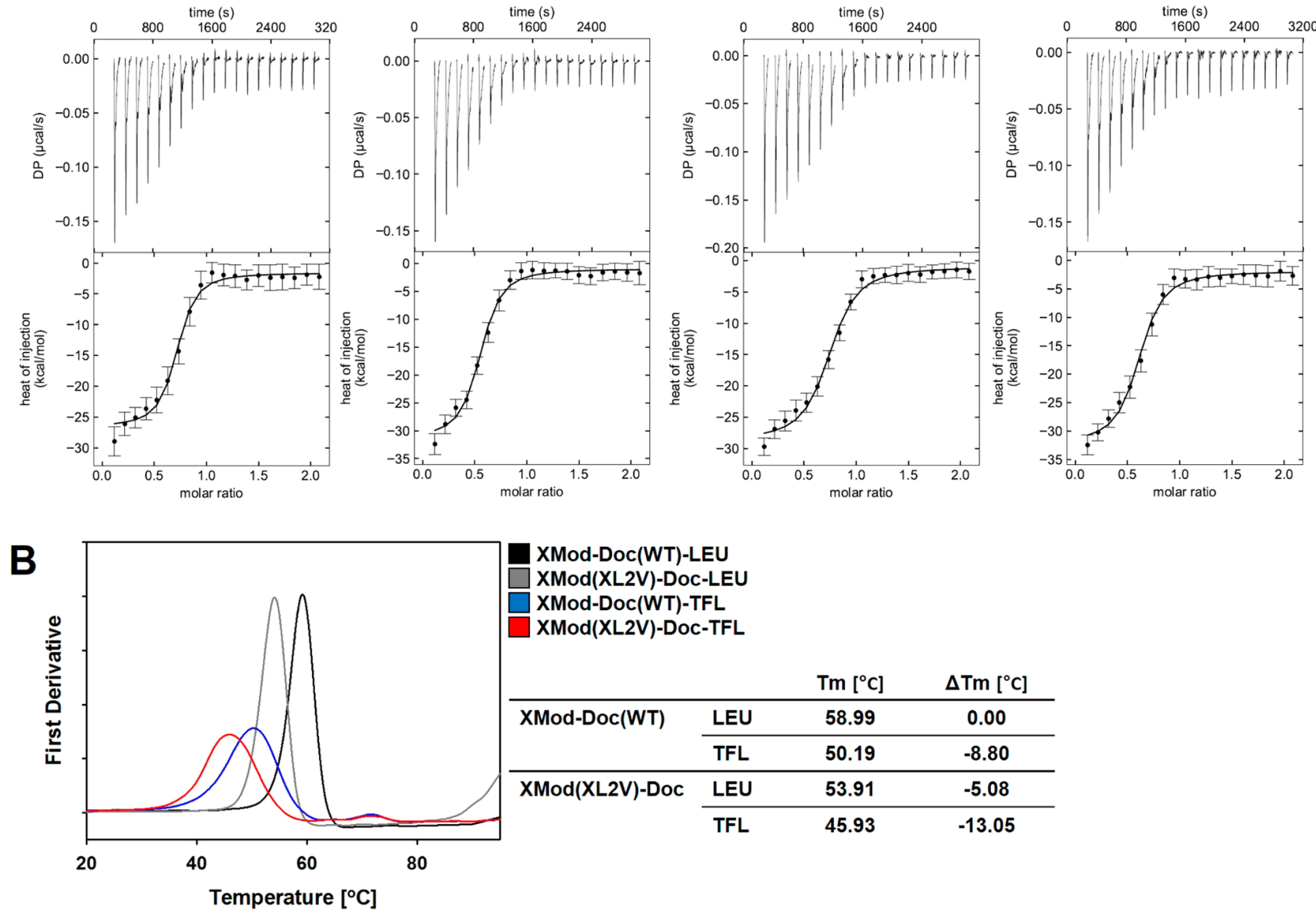

XMod-Doc(WT)-LEU

$X M o d(X L 2 V)$-Doc-LEU

XMod-Doc(WT)-TFL

$X M \operatorname{Mod}(X L 2 V)$-Doc-TFL

\begin{tabular}{lllc} 
& & $\operatorname{Tm}\left[{ }^{\circ} \mathrm{C}\right]$ & $\Delta \mathrm{Tm}\left[{ }^{\circ} \mathrm{C}\right]$ \\
\hline XMod-Doc(WT) & LEU & 58.99 & 0.00 \\
\cline { 2 - 4 } & TFL & 50.19 & -8.80 \\
\hline XMod(XL2V)-Doc & LEU & 53.91 & -5.08 \\
\cline { 2 - 4 } & TFL & 45.93 & -13.05
\end{tabular}

Figure 5. Analysis of bulk biophysical properties of XMod-Doc variants. (A) Binding affinity analyzed by ITC at equilibrium. (B) Thermal melting temperature of XMod-Doc variants measured by DSF.

$\mathrm{XMod}(\mathrm{XL2V})$-Doc-TFL is in contrast to the increase (7\%) in mechano-stability of XMod that we observed under TFL incorporation (Figure 4J) and confirms that non-equilibrium mechanical stability does not necessarily need to be correlated with enhanced thermal stability. ${ }^{30-33}$

Fluorination of proteins is used to tune biophysical properties, and its effects are well studied for systems at equilibrium. Here, for the first time we report the influence of fluorination on a molecular system under non-equilibrium mechanical tension. We investigated the influence of TFLincorporation on single-molecule unbinding and unfolding reactions within a mechano-stable XMod-Doc:Coh adhesion complex. We designed and produced LEU to VAL mutant variants which minimally disturbed native function of protein and allowed for TFL to be incorporated globally or only in Doc. Analysis of these variants using equilibrium biophysical stability assays and single-molecule AFM revealed several competing and counterintuitive effects.

First, we found that global fluorination of XMod-Doc(WT) changed the energy landscape of the Doc:Coh unbinding and XMod unfolding reactions. Fluorination tended to rigidify rupture/unfolding potentials for both WT and XL2V complexes, providing a steeper loading rate dependency (lower $\Delta x$ ), especially for P2 low-force rupture events and for P2 XMod unfolding events of XMod-Doc(WT). Second, global fluorination of XMod-Doc(WT) generated a new unbinding pathway (P3). We observed a clearly distinguishable rupture class that lacked XMod unfolding (Figure 3F) but ruptured at intermediate force. We attributed the emergence of this new pathway to alteration of the energy landscape by chirality or hydrophobicity of TFL. To gain more insight, we performed additional measurements at $\mathrm{pH} 5.5$ and found similar ratios between $\mathrm{P} 1, \mathrm{P} 2$, and $\mathrm{P} 3$ rupture events (Figure S7, Table S1). These dissociation pathways are therefore independent of $\mathrm{pH}$ across the range tested (5.5-7.2). Third and most significant, we found that when fluorination was localized to Doc, XMod was mechanically stabilized (Figure $4 \mathrm{~J})$. We consider this a counterintuitive finding given that the same sample was significantly less thermally stable (Figure 5B) and had little change in binding affinity (Figure 5A).

Taken together, these findings demonstrate how fluorination can modulate folding energy landscapes by lengthening or shortening binding/interaction potentials, generating new unbinding pathways, or even mechanically stabilizing adjacent non-fluorinated domains. These features highlight the orthogonality of mechanical and thermodynamic stability and provide new insights into the influence of fluorination on protein stability and function. This can broaden the 
applications of protein fluorination to include independent modulation of mechanical and thermodynamic stability of protein domains. With this work, we have provided a first look into how fluorination can regulate the mechanical properties of protein complexes and more generally protein-based biomaterials.

\section{ASSOCIATED CONTENT}

\section{SI Supporting Information}

The Supporting Information is available free of charge at https://pubs.acs.org/doi/10.1021/acs.nanolett.0c04178.

Details of the experimental materials and methods, AddGene plasmid numbers, supplementary figures (HRMS, size exclusion chromatography, SpyTag-SpyCatcher conjugation, AFM-SMFS at $\mathrm{pH}$ 5.5) and tables (percentages of rupture classes), and protein amino acid sequences (PDF)

\section{AUTHOR INFORMATION}

\section{Corresponding Author}

Michael A. Nash - Department of Chemistry, University of Basel, 4058 Basel, Switzerland; Department of Biosystems Science and Engineering, ETH Zurich, 4058 Basel, Switzerland; (1) orcid.org/0000-0003-3842-1567; Email: michael.nash@unibas.ch

\section{Authors}

Byeongseon Yang - Department of Chemistry, University of Basel, 4058 Basel, Switzerland; Department of Biosystems Science and Engineering, ETH Zurich, 4058 Basel, Switzerland

Haipei Liu - Department of Chemistry, University of Basel, 4058 Basel, Switzerland; Department of Biosystems Science and Engineering, ETH Zurich, 4058 Basel, Switzerland

Zhaowei Liu - Department of Chemistry, University of Basel, 4058 Basel, Switzerland; Department of Biosystems Science and Engineering, ETH Zurich, 4058 Basel, Switzerland

Regina Doenen - Department of Chemistry, University of Basel, 4058 Basel, Switzerland; Department of Biosystems Science and Engineering, ETH Zurich, 4058 Basel, Switzerland

Complete contact information is available at:

https://pubs.acs.org/10.1021/acs.nanolett.0c04178

\section{Notes}

The authors declare no competing financial interest.

\section{ACKNOWLEDGMENTS}

This work was supported by the University of Basel, ETH Zurich, an ERC Starting Grant (MMA-715207), the SNF NCCR in Molecular Systems Engineering, and the Swiss National Science Foundation (Project 200021_175478).

\section{REFERENCES}

(1) Wang, L.; Xie, J.; Schultz, P. G. Expanding the Genetic Code. Annu. Rev. Biophys. Biomol. Struct. 2006, 35, 225-249.

(2) Johnson, J. A.; Lu, Y. Y.; Van Deventer, J. A.; Tirrell, D. A. Residue-Specific Incorporation of Non-Canonical Amino Acids into Proteins: Recent Developments and Applications. Curr. Opin. Chem. Biol. 2010, 14 (6), 774-780.

(3) Lang, K.; Chin, J. W. Cellular Incorporation of Unnatural Amino Acids and Bioorthogonal Labeling of Proteins. Chem. Rev. 2014, 114 (9), 4764-4806.
(4) Mei, H.; Han, J.; Klika, K. D.; Izawa, K.; Sato, T.; Meanwell, N. A.; Soloshonok, V. A. Applications of Fluorine-Containing Amino Acids for Drug Design. Eur. J. Med. Chem. 2020, 186, 111826.

(5) Vulpetti, A.; Dalvit, C. Fluorine Local Environment: From Screening to Drug Design. Drug Discovery Today 2012, 17 (15-16), 890-897.

(6) Purser, S.; Moore, P. R.; Swallow, S.; Gouverneur, V. Fluorine in Medicinal Chemistry. Chem. Soc. Rev. 2008, 37 (2), 320-330.

(7) Robalo, J. R.; Huhmann, S.; Koksch, B.; Vila Verde, A. The Multiple Origins of the Hydrophobicity of Fluorinated Apolar Amino Acids. Chem. 2017, 3 (5), 881-897.

(8) Hoffmann, W.; Langenhan, J.; Huhmann, S.; Moschner, J.; Chang, R.; Accorsi, M.; Seo, J.; Rademann, J.; Meijer, G.; Koksch, B.; Bowers, M. T.; von Helden, G.; Pagel, K. An Intrinsic Hydrophobicity Scale for Amino Acids and Its Application to Fluorinated Compounds. Angew. Chem., Int. Ed. 2019, 58 (24), 8216-8220.

(9) Robalo, J. R.; Vila Verde, A. Unexpected Trends in the Hydrophobicity of Fluorinated Amino Acids Reflect Competing Changes in Polarity and Conformation. Phys. Chem. Chem. Phys. 2019, 21 (4), 2029-2038.

(10) Buer, B. C.; Marsh, E. N. G. Fluorine: A New Element in Protein Design. Protein Sci. 2012, 21 (4), 453-462.

(11) Panchenko, T.; Zhu, W. W.; Montclare, J. K. Influence of Global Fluorination on Chloramphenicol Acetyltransferase Activity and Stability. Biotechnol. Bioeng. 2006, 94 (5), 921-930.

(12) Holzberger, B.; Rubini, M.; Möller, H. M.; Marx, A. A Highly Active DNA Polymerase with a Fluorous Core. Angew. Chem., Int. Ed. 2010, 49 (7), 1324-1327.

(13) Berger, A. A.; Völler, J.-S.; Budisa, N.; Koksch, B. Deciphering the Fluorine Code - The Many Hats Fluorine Wears in a Protein Environment. Acc. Chem. Res. 2017, 50 (9), 2093-2103.

(14) Salwiczek, M.; Nyakatura, E. K.; Gerling, U. I. M.; Ye, S.; Koksch, B. Fluorinated Amino Acids: Compatibility with Native Protein Structures and Effects on Protein-protein Interactions. Chem. Soc. Rev. 2012, 41 (6), 2135-2171.

(15) Merkel, L.; Budisa, N. Organic Fluorine as a Polypeptide Building Element: In Vivo Expression of Fluorinated Peptides, Proteins and Proteomes. Org. Biomol. Chem. 2012, 10 (36), 72417261.

(16) Montclare, J. K.; Tirrell, D. A. Evolving Proteins of Novel Composition. Angew. Chem., Int. Ed. 2006, 45 (27), 4518-4521.

(17) Tang, Y.; Ghirlanda, G.; Petka, W. A.; Nakajima, T.; DeGrado, W. F.; Tirrell, D. A. Fluorinated Coiled-Coil Proteins Prepared in Vivo Display Enhanced Thermal and Chemical Stability. Angew. Chem., Int. Ed. 2001, 40 (8), 1494-1496.

(18) Tang, Y.; Tirrell, D. A. Biosynthesis of a Highly Stable CoiledCoil Protein Containing Hexafluoroleucine in an Engineered Bacterial Host. J. Am. Chem. Soc. 2001, 123 (44), 11089-11090.

(19) Tang, H.-C.; Lin, Y.-J.; Horng, J.-C. Modulating the Folding Stability and Ligand Binding Affinity of Pin 1 WW Domain by Proline Ring Puckering. Proteins: Struct., Funct., Genet. 2014, 82 (1), 67-76.

(20) Yoo, T. H.; Link, A. J.; Tirrell, D. A. Evolution of a Fluorinated Green Fluorescent Protein. Proc. Natl. Acad. Sci. U. S. A. 2007, 104 (35), 13887-13890.

(21) Dominguez, M. A., Jr; Thornton, K. C.; Melendez, M. G.; Dupureur, C. M. Differential Effects of Isomeric Incorporation of Fluorophenylalanines into PvuII Endonuclease. Proteins: Struct., Funct., Genet. 2001, 45 (1), 55-61.

(22) Parsons, J. F.; Xiao, G.; Gilliland, G. L.; Armstrong, R. N. Enzymes Harboring Unnatural Amino Acids: Mechanistic and Structural Analysis of the Enhanced Catalytic Activity of a Glutathione Transferase Containing 5-Fluorotryptophan. Biochemistry 1998, 37 (18), 6286-6294.

(23) Meng, H.; Kumar, K. Antimicrobial Activity and Protease Stability of Peptides Containing Fluorinated Amino Acids. J. Am. Chem. Soc. 2007, 129 (50), 15615-15622.

(24) Meng, H.; Krishnaji, S. T.; Beinborn, M.; Kumar, K. Influence of Selective Fluorination on the Biological Activity and Proteolytic 
Stability of Glucagon-like Peptide-1. J. Med. Chem. 2008, 51 (22), 7303-7307.

(25) Horng, J.-C.; Raleigh, D. P. Phi-Values beyond the Ribosomally Encoded Amino Acids: Kinetic and Thermodynamic Consequences of Incorporating Trifluoromethyl Amino Acids in a Globular Protein. J. Am. Chem. Soc. 2003, 125 (31), 9286-9287.

(26) Infante, E.; Stannard, A.; Board, S. J.; Rico-Lastres, P.; Rostkova, E.; Beedle, A. E. M.; Lezamiz, A.; Wang, Y. J.; Gulaidi Breen, S.; Panagaki, F.; Sundar Rajan, V.; Shanahan, C.; RocaCusachs, P.; Garcia-Manyes, S. The Mechanical Stability of Proteins Regulates Their Translocation Rate into the Cell Nucleus. Nat. Phys. 2019, 15 (9), 973-981.

(27) Rivas-Pardo, J. A.; Li, Y.; Mártonfalvi, Z.; Tapia-Rojo, R.; Unger, A.; Fernández-Trasancos, Á.; Herrero-Galán, E.; VelázquezCarreras, D.; Fernández, J. M.; Linke, W. A.; Alegre-Cebollada, J. A HaloTag-TEV Genetic Cassette for Mechanical Phenotyping of Proteins from Tissues. Nat. Commun. 2020, 11 (1), 2060.

(28) Lv, S.; Dudek, D. M.; Cao, Y.; Balamurali, M. M.; Gosline, J.; Li, H. Designed Biomaterials to Mimic the Mechanical Properties of Muscles. Nature 2010, 465 (7294), 69-73.

(29) Li, H.; Cao, Y. Protein Mechanics: From Single Molecules to Functional Biomaterials. Acc. Chem. Res. 2010, 43 (10), 1331-1341. (30) Li, H.; Oberhauser, A. F.; Fowler, S. B.; Clarke, J.; Fernandez, J. M. Atomic Force Microscopy Reveals the Mechanical Design of a Modular Protein. Proc. Natl. Acad. Sci. U. S. A. 2000, 97 (12), 65276531.

(31) Oroz, J.; Bruix, M.; Laurents, D. V.; Galera-Prat, A.; Schönfelder, J.; Cañada, F. J.; Carrión-Vázquez, M. The Y9P Variant of the Titin I27 Module: Structural Determinants of Its Revisited Nanomechanics. Structure 2016, 24 (4), 606-616.

(32) Oberhauser, A. F.; Badilla-Fernandez, C.; Carrion-Vazquez, M.; Fernandez, J. M. The Mechanical Hierarchies of Fibronectin Observed with Single-Molecule AFM. J. Mol. Biol. 2002, 319 (2), 433-447.

(33) Best, R. B.; Li, B.; Steward, A.; Daggett, V.; Clarke, J. Can NonMechanical Proteins Withstand Force? Stretching Barnase by Atomic Force Microscopy and Molecular Dynamics Simulation. Biophys. J. 2001, 81 (4), 2344-2356.

(34) Ott, W.; Jobst, M. A.; Schoeler, C.; Gaub, H. E.; Nash, M. A. Single-Molecule Force Spectroscopy on Polyproteins and Receptorligand Complexes: The Current Toolbox. J. Struct. Biol. 2017, 197 (1), 3-12.

(35) Chen, Y.; Radford, S. E.; Brockwell, D. J. Force-Induced Remodelling of Proteins and Their Complexes. Curr. Opin. Struct. Biol. 2015, 30, 89-99.

(36) Schönfelder, J.; De Sancho, D.; Perez-Jimenez, R. The Power of Force: Insights into the Protein Folding Process Using SingleMolecule Force Spectroscopy. J. Mol. Biol. 2016, 428 (21), 42454257.

(37) Nathwani, B.; Shih, W. M.; Wong, W. P. Force Spectroscopy and Beyond: Innovations and Opportunities. Biophys. J. 2018, 115 (12), 2279-2285.

(38) Jacobson, D. R.; Uyetake, L.; Perkins, T. T. Membrane-Protein Unfolding Intermediates Detected with Enhanced Precision Using a Zigzag Force Ramp. Biophys. J. 2019, 118 (3), 667-675.

(39) Yu, H.; Siewny, M. G. W.; Edwards, D. T.; Sanders, A. W.; Perkins, T. T. Hidden Dynamics in the Unfolding of Individual Bacteriorhodopsin Proteins. Science 2017, 355 (6328), 945-950.

(40) Dietz, H.; Berkemeier, F.; Bertz, M.; Rief, M. Anisotropic Deformation Response of Single Protein Molecules. Proc. Natl. Acad. Sci. U. S. A. 2006, 103 (34), 12724-12728.

(41) Sedlak, S. M.; Schendel, L. C.; Gaub, H. E.; Bernardi, R. C. Streptavidin/biotin: Tethering Geometry Defines Unbinding Mechanics. Sci. Adv. 2020, 6 (13), eaay5999.

(42) Sedlak, S. M.; Schendel, L. C.; Melo, M. C. R.; Pippig, D. A.; Luthey-Schulten, Z.; Gaub, H. E.; Bernardi, R. C. Direction Matters: Monovalent Streptavidin/Biotin Complex under Load. Nano Lett. 2019, 19 (6), 3415-3421.
(43) Carrion-Vazquez, M.; Li, H.; Lu, H.; Marszalek, P. E.; Oberhauser, A. F.; Fernandez, J. M. The Mechanical Stability of Ubiquitin Is Linkage Dependent. Nat. Struct. Mol. Biol. 2003, 10 (9), $738-743$.

(44) Brockwell, D. J.; Paci, E.; Zinober, R. C.; Beddard, G. S.; Olmsted, P. D.; Smith, D. A.; Perham, R. N.; Radford, S. E. Pulling Geometry Defines the Mechanical Resistance of a Beta-Sheet Protein. Nat. Struct. Mol. Biol. 2003, 10 (9), 731-737.

(45) Jobst, M. A.; Milles, L. F.; Schoeler, C.; Ott, W.; Fried, D. B.; Bayer, E. A.; Gaub, H. E.; Nash, M. A. Resolving Dual Binding Conformations of Cellulosome Cohesin-Dockerin Complexes Using Single-Molecule Force Spectroscopy. eLife 2015, 4, e10319.

(46) Nash, M. A.; Smith, S. P.; Fontes, C. M.; Bayer, E. A. Single versus Dual-Binding Conformations in Cellulosomal Cohesindockerin Complexes. Curr. Opin. Struct. Biol. 2016, 40, 89-96.

(47) Bogan, A. A.; Thorn, K. S. Anatomy of Hot Spots in Protein Interfaces. J. Mol. Biol. 1998, 280 (1), 1-9.

(48) Voronov-Goldman, M.; Yaniv, O.; Gul, O.; Yoffe, H.; SalamaAlber, O.; Slutzki, M.; Levy-Assaraf, M.; Jindou, S.; Shimon, L. J. W.; Borovok, I.; Bayer, E. A.; Lamed, R.; Frolow, F. Standalone Cohesin as a Molecular Shuttle in Cellulosome Assembly. FEBS Lett. 2015, 589 (14), 1569-1576.

(49) Schoeler, C.; Malinowska, K. H.; Bernardi, R. C.; Milles, L. F.; Jobst, M. A.; Durner, E.; Ott, W.; Fried, D. B.; Bayer, E. A.; Schulten, K.; Gaub, H. E.; Nash, M. A. Ultrastable Cellulosome-Adhesion Complex Tightens under Load. Nat. Commun. 2014, 5, 5635.

(50) Schoeler, C.; Bernardi, R. C.; Malinowska, K. H.; Durner, E.; Ott, W.; Bayer, E. A.; Schulten, K.; Nash, M. A.; Gaub, H. E. Mapping Mechanical Force Propagation through Biomolecular Complexes. Nano Lett. 2015, 15 (11), 7370-7376.

(51) Bernardi, R. C.; Durner, E.; Schoeler, C.; Malinowska, K. H.; Carvalho, B. G.; Bayer, E. A.; Luthey-Schulten, Z.; Gaub, H. E.; Nash, M. A. Mechanisms of Nanonewton Mechanostability in a Protein Complex Revealed by Molecular Dynamics Simulations and SingleMolecule Force Spectroscopy. J. Am. Chem. Soc. 2019, 141 (37), 14752-14763.

(52) Verdorfer, T.; Bernardi, R. C.; Meinhold, A.; Ott, W.; LutheySchulten, Z.; Nash, M. A.; Gaub, H. E. Combining in Vitro and in Silico Single-Molecule Force Spectroscopy to Characterize and Tune Cellulosomal Scaffoldin Mechanics. J. Am. Chem. Soc. 2017, 139 (49), 17841-17852.

(53) Liu, Z.; Liu, H.; Vera, A. M.; Bernardi, R. C.; Tinnefeld, P.; Nash, M. A. High Force Catch Bond Mechanism of Bacterial Adhesion in the Human Gut. Nat. Commun. 2020, 11, 4321.

(54) Jindou, S.; Brulc, J. M.; Levy-Assaraf, M.; Rincon, M. T.; Flint, H. J.; Berg, M. E.; Wilson, M. K.; White, B. A.; Bayer, E. A.; Lamed, R; Borovok, I. Cellulosome Gene Cluster Analysis for Gauging the Diversity of the Ruminal Cellulolytic Bacterium Ruminococcus Flavefaciens. FEMS Microbiol. Lett. 2008, 285 (2), 188-194.

(55) Ding, S. Y.; Rincon, M. T.; Lamed, R.; Martin, J. C.; McCrae, S. I.; Aurilia, V.; Shoham, Y.; Bayer, E. A.; Flint, H. J. Cellulosomal Scaffoldin-Like Proteins fromRuminococcus Flavefaciens. J. Bacteriol. 2001, 183 (6), 1945-1953.

(56) Salama-Alber, O.; Jobby, M. K.; Chitayat, S.; Smith, S. P.; White, B. A.; Shimon, L. J. W.; Lamed, R.; Frolow, F.; Bayer, E. A. Atypical Cohesin-Dockerin Complex Responsible for Cell Surface Attachment of Cellulosomal Components: BINDING FIDELITY, PROMISCUITY, AND STRUCTURAL BUTTRESSES. J. Biol. Chem. 2013, 288 (23), 16827-16838.

(57) Montclare, J. K.; Son, S.; Clark, G. A.; Kumar, K.; Tirrell, D. A. Biosynthesis and Stability of Coiled-Coil Peptides Containing (2 S ,4 $\mathrm{R}$ ) -5,5,5-Trifluoroleucine and (2 $\mathrm{S}, 4 \mathrm{~S}$ )-5,5,5-Trifluoroleucine. ChemBioChem 2009, 10 (1), 84-86.

(58) Liu, H.; Ta, D. T.; Nash, M. A. Mechanical Polyprotein Assembly Using Sfp and Sortase-Mediated Domain Oligomerization for Single-Molecule Studies. Small Methods 2018, 2 (6), 1800039.

(59) Liu, H.; Schittny, V.; Nash, M. A. Removal of a Conserved Disulfide Bond Does Not Compromise Mechanical Stability of a VHH Antibody Complex. Nano Lett. 2019, 19 (8), 5524-5529. 
(60) Yang, B.; Liu, Z.; Liu, H.; Nash, M. A. Next Generation Methods for Single-Molecule Force Spectroscopy on Polyproteins and Receptor-Ligand Complexes. Frontiers in Molecular Biosciences 2020, 7, 85 .

(61) Wieduwild, R.; Howarth, M. Assembling and Decorating Hyaluronan Hydrogels with Twin Protein Superglues to Mimic CellCell Interactions. Biomaterials 2018, 180, 253-264.

(62) Zakeri, B.; Howarth, M. Spontaneous Intermolecular Amide Bond Formation between Side Chains for Irreversible Peptide Targeting. J. Am. Chem. Soc. 2010, 132 (13), 4526-4527.

(63) Zakeri, B.; Fierer, J. O.; Celik, E.; Chittock, E. C.; SchwarzLinek, U.; Moy, V. T.; Howarth, M. Peptide Tag Forming a Rapid Covalent Bond to a Protein, through Engineering a Bacterial Adhesin. Proc. Natl. Acad. Sci. U. S. A. 2012, 109 (12), E690-E697.

(64) Schwaiger, I.; Kardinal, A.; Schleicher, M.; Noegel, A. A.; Rief, M. A Mechanical Unfolding Intermediate in an Actin-Crosslinking Protein. Nat. Struct. Mol. Biol. 2004, 11 (1), 81-85.

(65) Ott, W.; Jobst, M. A.; Bauer, M. S.; Durner, E.; Milles, L. F.; Nash, M. A.; Gaub, H. E. Elastin-like Polypeptide Linkers for SingleMolecule Force Spectroscopy. ACS Nano 2017, 11 (6), 6346-6354.

(66) Yin, J.; Straight, P. D.; McLoughlin, S. M.; Zhou, Z.; Lin, A. J.; Golan, D. E.; Kelleher, N. L.; Kolter, R.; Walsh, C. T. Genetically Encoded Short Peptide Tag for Versatile Protein Labeling by Sfp Phosphopantetheinyl Transferase. Proc. Natl. Acad. Sci. U. S. A. 2005, 102 (44), 15815-15820.

(67) Baba, T.; Ara, T.; Hasegawa, M.; Takai, Y.; Okumura, Y.; Baba, M.; Datsenko, K. A.; Tomita, M.; Wanner, B. L.; Mori, H. Construction of Escherichia Coli K-12 in-Frame, Single-Gene Knockout Mutants: The Keio Collection. Mol. Syst. Biol. 2006, 2 (1), 2006.0008.

(68) Yang, B.; Ayyadurai, N.; Yun, H.; Choi, Y. S.; Hwang, B. H.; Huang, J.; Lu, Q.; Zeng, H.; Cha, H. J. In Vivo Residue-Specific Dopa-Incorporated Engineered Mussel Bioglue with Enhanced Adhesion and Water Resistance. Angew. Chem., Int. Ed. 2014, 53 (49), 13360-13364.

(69) Evans, E.; Ritchie, K. Dynamic Strength of Molecular Adhesion Bonds. Biophys. J. 1997, 72 (4), 1541-1555.

(70) Merkel, R.; Nassoy, P.; Leung, A.; Ritchie, K.; Evans, E. Energy Landscapes of Receptor-ligand Bonds Explored with Dynamic Force Spectroscopy. Nature 1999, 397 (6714), 50-53.

(71) Brockwell, D. J.; Beddard, G. S.; Paci, E.; West, D. K.; Olmsted, P. D.; Smith, D. A.; Radford, S. E. Mechanically Unfolding the Small, Topologically Simple Protein L. Biophys. J. 2005, 89 (1), 506-519.

(72) Schoeler, C.; Verdorfer, T.; Gaub, H. E.; Nash, M. A. Biasing Effects of Receptor-Ligand Complexes on Protein-Unfolding Statistics. Phys. Rev. E: Stat. Phys., Plasmas, Fluids, Relat. Interdiscip. Top. 2016, 94 (4-1), No. 042412. 